\title{
Commentary \\ Self-calibrating pulse contour cardiac output: do validation studies really show its clinical reliability?
}

Lester AH Critchley

Department of Anaesthesia and Intensive Care, The Chinese University of Hong Kong, Prince of Wales Hospital, Shatin, New Territories, Hong Kong, China

Corresponding author: Lester AH Critchley, hcritchley@cuhk.edu.hk

Published: 4 March 2009

This article is online at http://ccforum.com/content/13/2/123

(c) 2009 BioMed Central Ltd

See related research by Senn et al., http://ccforum.com/content/13/2/R32
Critical Care 2009, 13:123 (doi:10.1186/cc7718)

\begin{abstract}
The present study was performed to test a new software version of the FloTrac/Vigileo using head-up-head-down tilting in postcardiac surgery patients. Impressive improvements in Bland and Altman limits of agreement from $37.5 \%$ to $21.6 \%$ were recorded. The results, however could be attributed to a failure to produce a wide enough range of test circulatory conditions. A more rigorous test of performance is needed before any real conclusion concerning use of the FloTrac/Vigileo in clinical practice can be made.
\end{abstract}

In the current issue Senn and colleagues present their findings [1] from a study aimed at validating a new version of the FloTrac/Vigileo monitor (Edward Lifesciences, Irvine, CA, USA), a system that measures cardiac output using the arterial pulse contour method.

The pulse contour method is relatively new on the cardiac output measurement scene. The method was first used commercially in the Finapres (Ohmedia, Englewood, CO, USA), a device that used a pneumatic finger cuff to continuously measure blood pressure. By transforming the pressure wave into a flow wave, cardiac output was measured from the area under the flow curve.

In the past few years a number of pulse contour methods based on the arterial line pressure trace have been developed. The most well known of these methods are the PiCCO (Pulsion Medical Systems, Munich, Germany), the LiDCO (LiDCO Group Plc, London, UK) and the FloTrac/Vigileo. Until recently these systems have all required prior calibration using a second method of cardiac output measurement, either thermodilution (pulmonary artery or transpulmonary) or lithium dye dilution. The FloTrac/Vigileo has recently developed a method of self-calibration based on imputing patient demographic data.

The main concern with all pulse contour systems has been their failure to properly adjust to changes in circulatory dynamics, such as blood loss or peripheral vasoconstriction $[2,3]$. This failure has lead to more favourable reviews when these systems have been validated in the relatively stable haemodynamic setting of postcardiac surgery, but to less favourable reviews when validated in septic or liver transplant patients, where the circulatory changes are more challenging [3].

The points to note about Senn and colleagues' study are that updated software (version 1.07) was evaluated, by comparing 25 patients using the old software (version 1.03) and 25 patients using the new software (version 1.07). Comparisons of the FloTrac/Vigileo were also made against pulse contour and transpulmonary cardiac outputs using the Pulsion PiCCO system. Three serial changes in cardiac output were generated by head-up-head-down tilting, and the trending ability was assessed. Patients were kept haemodynamically stable throughout the study with propofol-remifentanil sedation, a pacing wire to control the heart rate and noradrenaline infusion targeted at a mean arterial blood pressure of $70 \mathrm{mmHg}$.

Their data showed a marked improvement in Bland and Altman limits of agreement between set A data (37.5\%) and set B data (21.6\%). The limits for the PiCCO, however, were equally small (25.5\%). There was also a $20 \%$ difference in the reference cardiac output between the sets following the head-down tilt, as the set $A$ reference output was $6.1 \mathrm{l} / \mathrm{min}$ and the set $B$ reference output was $5.0 \mathrm{l} / \mathrm{min}$. The last observation is relevant because it suggests the two sets of patients were haemodynamically different in respect to tilting responses. Differences in nursing management and the use of fluids, sedation and noradrenaline in the intensive care unit could have been responsible for this difference.

The improvement in Bland and Altman limits of agreement from $37.5 \%$ to $21.6 \%$ is puzzling. Judged against the $\pm 28.3 \%$ acceptable level, the new software version 
performed very reliably [4] - but so did the PiCCO, with limits of $\pm 25.5 \%$. The authors attributed this improvement to more frequent recalibration from 10 minutes to 1 minute. Readings in the study were taken after 15 minutes of continuous tilt, however, so recalibration should have occurred with both software versions. Furthermore, the basic algorithm to adjust for circulatory changes does not seem to have been modified. More recent software (version 1.10) still did not cope with the marked changes in systemic vascular resistance seen in liver transplant patients [3].

These impressive agreement statistics may reflect experimental design and a failure to generate sufficiently large changes in haemodynamics to properly test the device. In set $B$ data the systemic vascular resistance only changed by $5 \%$ (from 960 to $1,008 \mathrm{dyn} \cdot \mathrm{s} \cdot \mathrm{cm}^{-5}$ ). Head-up tilting to $30^{\circ}$ should produce at least a $10 \%$ to $20 \%$ increase in peripheral resistance in healthy adults [5]. Sedation and controlling the circulation with noradrenaline may have annulled normal responses to tilting.

Senn and colleagues' study does not in my opinion comprehensively test the FloTrac/Vigileo system as their use of tilting did not provoke sufficiently large changes in haemodynamics [1]. Little new information can therefore be gained about the reliability of the FloTrac/Vigileo, other than the new software only improving reliability by more frequent recalibration.

The FloTrac/Vigileo system has yet to be shown to cope with more extreme circulatory conditions. New software versions need to be rigorously tested in the animal laboratory against a true reference standard, such as an aortic flow probe, and need to be shown to track changes in cardiac output reliably over a range of conditions. Only then should human studies be performed against thermodilution, using the standard Bland and Altman approach. Finally, one should not forget that the clinical utility of the device would still need to be shown in randomized, clinical controlled trials.

\section{Competing interests}

The author declares that they have no competing interests.

\section{References}

1. Senn A, Button D, Zollinger A, Hofer CK: Assessment of cardiac output changes using a modified FloTrac/Vigileo ${ }^{\mathrm{TM}}$ algorithm in cardiac surgery patients. Crit Care 2009, 13:R32.

2. Bein B, Meybohm P, Cavus E, Renner J, Tonner PH, Steinfath M, Scholz J, Doerges V: The reliability of pulse contour-derived cardiac output during hemorrhage and after vasopressor administration. Anesth Analg 2007, 105:107-113.

3. Biancofiore G, Critchley LA, Lee A, Bindi L, Bisà M, Esposito M, Meacci L, Mozzo R, DeSimone P, Urbani L, Filipponi F: Evaluation of an uncalibrated arterial pulse contour cardiac output monitoring system in cirrhotic patients undergoing liver surgery. $\mathrm{Br}$ $J$ Anaesth 2009, 102:47-54.

4. Critchley LA, Critchley JA: A meta-analysis of studies using bias and precision statistics to compare cardiac output measurement techniques. J Clin Monit Comput 1999, 15:85-91.

5. Critchley LA, Conway F, Anderson PJ, Tomlinson B, Critchley JA:
Non-invasive continuous arterial pressure, heart rate and stroke volume measurements during graded head-up tilt in normal man. Clin Auton Res 1997, 7:97-101. 